\title{
Análise das Tensões Residuais Utilizando o Método DPC na Soldagem Multipasse do Aço ASTM A131 AH36 pelos Processos SMAW e FCAW
}

\author{
Danilo Helder de Melo Pereira ${ }^{1}$ (D), Douglas Henrique de Melo Pereira ${ }^{1}$ (D), Tiago Leite Rolim $^{1}$ (D), Ricardo Artur Sanguinetti Ferreira ${ }^{1}$ (D) \\ ${ }^{1}$ Universidade Federal de Pernambuco - UFPE, Recife, PE, Brasil.
}

Como citar: Pereira DHM, Pereira DHM, Rolim TL, Ferreira RAS. Análise das tensões residuais utilizando o método DPC na soldagem multipasse do aço ASTM A131 AH36 pelos processos SMAW e FCAW. Soldagem \& Inspeção. 2020;25:e2513. https://doi.org/10.1590/01049224/SI25.13

\begin{abstract}
Resumo: As tensões residuais (TR) nas juntas soldadas do aço ASTM A131 AH36 foram analisadas pelo método DPC (deslocamento de pontos coordenados). A soldagem a arco foi realizada pelos processos eletrodo revestido (ER) e arame tubular (AT). As chapas de teste com dimensões de $200 \mathrm{~mm}$ x $65 \mathrm{~mm}$ e espessura 31,75 mm foram soldadas, segundo as normas (fabricação e qualificação) AWS D1.1 [1] e Petrobrás N-133 [2], respectivamente. Depois de soldadas foram realizados furos equidistantes com diâmetros de 2,00 $\mathrm{mm}$ por 2,00 $\mathrm{mm}$ de profundidade ao longo da zona termicamente afetada. Após a furação, tais furos foram referenciados em uma mesa de medição por coordenadas. Em seguida, as chapas de teste soldadas foram submetidas a um tratamento térmico para o alívio de tensões (TTAT) e, em seguida, foram medidos os deslocamentos dos furos causados pelo tratamento, o que possibilitou o cálculo das tensões residuais. Os resultados obtidos mostraram uma boa correlação do DPC com outros métodos de medições das TR.
\end{abstract}

Palavras-chave: Deslocamento de pontos coordenados; Método DPC; Tensões residuais.

\section{Residual stress analysis using CPD method in ASTM A131 AH36 steel multipass welding by SMAW and FCAW processes}

\begin{abstract}
The residual stress (RS) in welded joints of ASTM A131 AH36 were analyzed by the method DPC (coordinated points displacement). The arc welding was performed using by Shielded Metal Arc Welding (SMAW) and Flux Cored Arc Welding (FCAW) processes. The test plates with dimensions of $200 \mathrm{~mm} \times 65 \mathrm{~mm}$ and thickness of 31,75 mm were welded according to AWS D1.1 [1] and Petrobras N-133 [2] standards (manufacture and qualification), respectively. After welding, equidistant holes with diameters of $2.00 \mathrm{~mm}$ and depth of $2.00 \mathrm{~mm}$ were drilled along the heat affected zone. After drilling, the holes were referenced on a coordinate measuring table. Then, the welded test plates were subjected to Post Weld Heat Treatment (PWHT) and then the displacements of the holes caused by the treatment were measured, making possible the calculation of residual stresses. The results obtained showed a good correlation of the DPC with other methods of measurement for TR.
\end{abstract}

Key-words: Coordinate Point Difference; DPC Method; Residual Stresses.

\section{Introdução}

Na engenharia de soldagem, é de suma importância a seleção adequada do processo e do procedimento de soldagem a ser utilizado com relação aos custos, qualidade e tempo de fabricação. O processo de soldagem com eletrodos revestidos é o mais utilizado no mundo, principalmente devido a sua simplicidade, versatilidade, qualidade das soldas e baixo custo de equipamentos e consumíveis. Numa soldagem semiautomática, o processo com Arame Tubular (AT) é o que permite maiores velocidades de soldagem devido às maiores taxas de deposição alcançadas, aumentando, assim, a produtividade da soldagem. Além disso, possui um arco mais largo, distribuindo a energia de soldagem mais uniformemente. Sua penetração, por ter característica mais rasa e larga, tende a reduzir os riscos de falta de fusões, minimizando a probabilidade de custos e o tempo com retrabalhos e novas inspeções.

A segurança, a produtividade e a competitividade do mercado tornaram cada vez mais necessário o estudo do estado de fabricação e a manutenção dos equipamentos. Um desses indicadores são as tensões residuais, que podem induzir falhas e redução de vida útil deles. $O$ estudo das tensões residuais se torna fundamental nos materiais que foram submetidos à variação de temperatura não uniforme. Segundo Macherauch e Kloos [1], as tensões residuais "são tensões auto equilibradas existentes nos materiais na ausência de esforços externo, deslocamentos ou gradientes de temperatura". 
O nível de tensões residuais pode ser analisado para a qualificação dos processos de fabricação que introduzem tensões residuais no material, como o jateamento, o granalhamento, a soldagem, a fundição, a conformação, a usinagem e os tratamentos térmicos [2]. A previsão de tensões residuais em processos de soldagem, a partir de modelos analíticos e numéricos, ainda são imprecisos devido à inexistência de valores de propriedades termofísicas e mecânicas de diferentes aços em função da temperatura. Diante dessa realidade, os métodos experimentais se tornam uma ferramenta indispensável aos estudos.

Na presente análise, as tensões residuais foram medidas pela metodologia DPC em Siqueira Filho et al. [3] que, conforme será mostrado, é de baixo custo e de fácil aplicação conforme Melo et al. [4] e sensível aos efeitos da anisotropia Sugahara et al. [5]. Isso pode ampliar a sua gama de aplicações devido aos bons resultados obtidos e excelente custo benefício para a análise de tensões residuais.

\section{Materiais e Métodos}

\subsection{Metal de base}

O metal de base utilizado neste estudo foi o ASTM 131 AH36 [6], um aço estrutural usado na indústria naval, no qual o grau determina as condições de materiais e fabricação, composição química e requisitos mecânicos. As chapas foram cedidas por uma empresa que presta serviços a um estaleiro na região, cuja composição química foi analisada por espectrometria óptica em um laboratório acreditado pelo IMETRO, conforme se pode observar na Tabela 1.

Tabela 1. Composição química do metal de base.

\begin{tabular}{cccccccccc}
\hline \multicolumn{7}{c}{ Análise Química via Espectometria - Óptica (em porcentagem) } \\
\hline $\mathbf{C}$ & $\mathbf{S i}$ & $\mathbf{M n}$ & $\mathbf{P}$ & $\mathbf{S}$ & $\mathbf{N i}$ & $\mathbf{M o}$ & $\mathbf{C u}$ & $\mathbf{A l}$ \\
0,1000 & 0,2830 & 1,6000 & 0,0360 & 0,0200 & 0,0130 & 0,0040 & 0,1660 & 0,0240 \\
$\mathrm{~V}$ & $\mathrm{Nb}$ & $\mathrm{Ti}$ & $\mathrm{Pb}$ & $\mathrm{Sn}$ & $\mathrm{Co}$ & $\mathrm{As}$ & $\mathrm{B}$ & $\mathrm{Fe}$ & \\
0,0370 & 0,0360 & 0,0150 & 0,0010 & 0,0010 & 0,0030 & 0,0030 & 0,0004 & BASE \\
\hline
\end{tabular}

Os corpos de prova foram cortados em serra de fita com as dimensões de $200 \mathrm{~mm} \times 65 \mathrm{~mm} \times 31,75 \mathrm{~mm}$, chanfradas com ângulo de $25^{\circ}$ e, posteriormente, montados em pares, com uma abertura de raiz de $2 \mathrm{~mm}$, fixadas a duas chapas de $12,7 \mathrm{~mm}$, como dispositivo auxiliar de montagem, conforme a Figura 1.

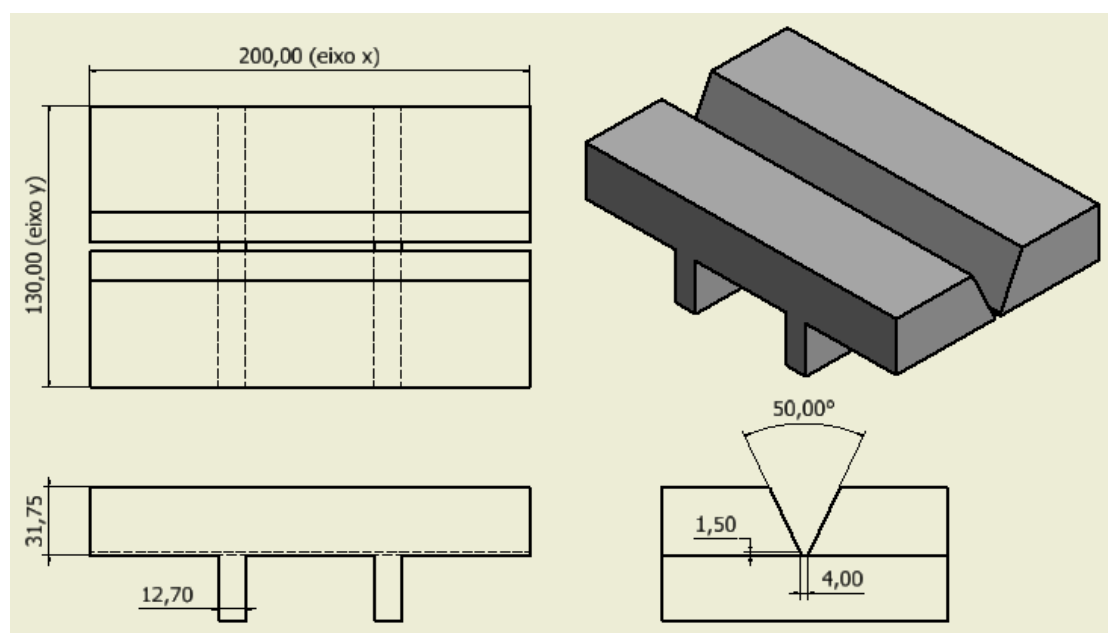

Figura 1. Desenho técnico do corpo de prova.

\subsection{Consumíveis de soldagem}

Os consumíveis de soldagem utilizados nesta pesquisa são homologados pela Fundação Brasileira de Tecnologia da Soldagem (FBTS). O eletrodo é revestido de especificação AWS SFA 5.1 [7] e classificação E-7018, diâmetro de 3,25 mm. A escolha desse eletrodo advém das suas características em produzir o metal de solda com resistência mínima de 485 MPa e por ser o eletrodo de baixo hidrogênio mais consolidado no mercado. O arame tubular foi utilizado para a soldagem em conformidade com a especificação AWS SFA 5.20 [8] de classificação ER 71T-1 auto protegido e de diâmetro 1,2 mm. A resistência à tração 
mínima do seu metal de solda é de $485 \mathrm{Mpa}$. O tratamento e armazenamento dos consumíveis foram realizados conforme as recomendações do fabricante e norma Petrobrás N-133 [9].

\subsection{Fontes de soldagem}

As máquinas de soldagem foram devidamente calibradas e utilizadas em rede trifásica 380V. Para o processo de eletrodo revestido, foi utilizado uma retificadora de tensão em vazio $75 \mathrm{~V}$, faixa de correntes de 50 a $500 \mathrm{~A}$, potência máxima 36 kVA e fator de potência 0,6 . No processo arame tubular, a tensão em vazio foi de 16 a $52 \mathrm{~V}$, tensão de trabalho de 15,5 a $39 \mathrm{~V}$ com faixa de correntes de 50 a 500 A, potência máxima foi de 22,7 kVA e o fator de potência de 0,8 .

\subsection{Procedimento de soldagem}

As soldagens foram realizadas conforme os procedimentos de soldagem EPS 001/19 e EPS 005/19, qualificadas conforme norma AWS D1.1 [10] e recomendações Petrobrás N-133 [9]. Em ambos os processos, as soldas em juntas de topo com bisel de $50^{\circ}$, posição de soldagem $1 \mathrm{G}$, foram executadas por soldador qualificado e todos os corpos de prova obtiveram boa qualidade de acabamento e ensaios visuais de soldas aprovados. Todos os passes foram dados na mesma direção e finalizando com goivagem, ensaio por líquido penetrante, para garantir a remoção adequada, limpeza e realização da contra solda na raiz (passe 10), conforme se pode verificar na seguinte sequência de soldagem.

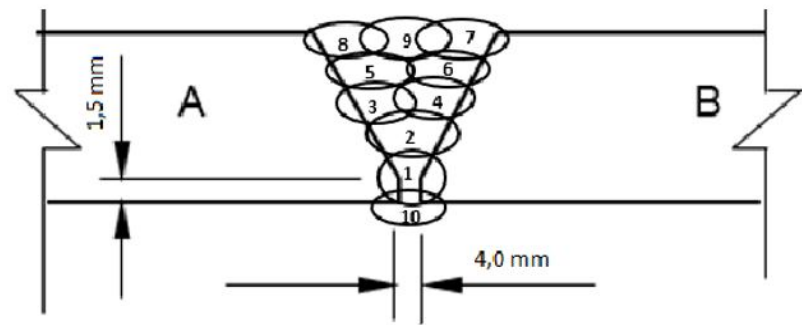

Figura 2. (a) Sequência de soldagem (b) Montagem do corpo de prova.

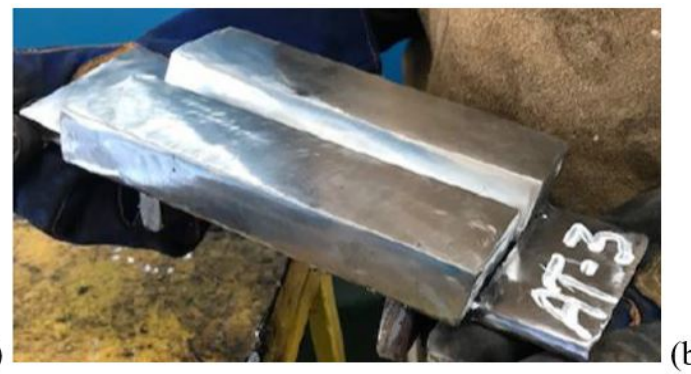

(b)

Inicialmente, as juntas foram ponteadas, soldadas às chapas apêndices para a abertura e o fechamento do arco elétrico e depois foram soldadas às chapas auxiliares de montagem de $12,7 \mathrm{~mm}$ de espessura para restringirem as deformações (ver Figura 2b). Não houve pré-aquecimento e a temperatura de interpasse máxima permitida foi de $250{ }^{\circ} \mathrm{C}$. Os parâmetros de soldagem de ambos os processos foram extraídos das respectivas EPS já qualificadas. Foi inspecionado e registrado os parâmetros de cada passe e para posterior determinação de energia de soldagem, sendo a velocidades da soldagem medida pela razão do comprimento do passe pelo tempo de sua execução (Tabela 1). A qualificação dos procedimentos utilizados, assim como o soldador, foi realizada por inspetor de soldagem N2, conforme as normas aplicáveis. Na Figura 3 a seção do corpo de prova soldado.

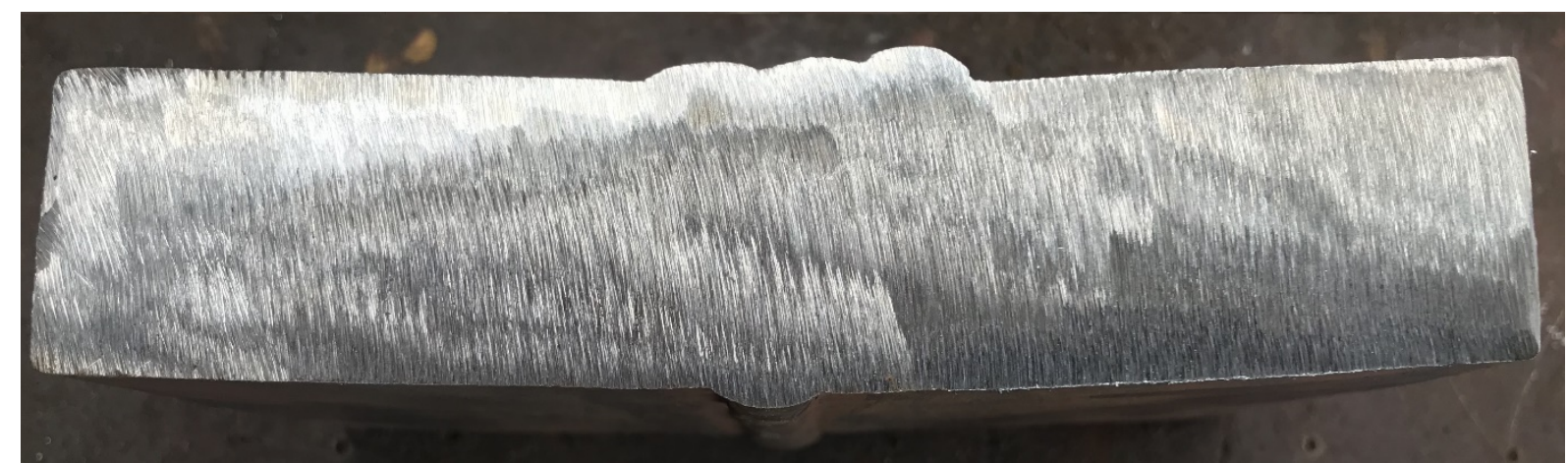

Figura 3. Seção do corpo de prova soldado.

\subsection{Cálculo da energia de soldagem}

Baseado nos dados obtidos durante o acompanhamento das soldagens, foi calculada a energia de soldagem de cada corpo de prova, conforme a Equação 1, resultados mostrados na Tabela 2. 
$E=f x \frac{V x I}{v}$

Tabela 2. Parâmetros para cálculo da Energia de Soldagem.

\begin{tabular}{ccccccc}
\hline Parâmetros médios & ER-01 & ER-02 & ER-03 & AT-01 & AT-02 & AT-03 \\
Eficiência térmica (f) & 0,8 & 0,8 & 0,8 & 0,8 & 0,8 & 0,8 \\
Tensão (V) & 22,9 & 23,1 & 23,1 & 27,18 & 26,36 & 27,09 \\
Corrente (A) & 138,1 & 138,4 & 137,7 & 195,91 & 190,18 & 192,91 \\
Vel. Soldagem (v) $[\mathrm{mm} / \mathrm{min}]$ & 66,96 & 68,72 & 66,36 & 202,01 & 207,49 & 197,10 \\
Energia de Soldagem (E) $[\mathrm{KJ} / \mathrm{mm}]$ & 2,31 & 2,29 & 2,34 & 1,3 & 1,21 & 1,33 \\
\hline
\end{tabular}

\subsection{Marcação e furação dos pontos e medição por coordenadas}

Cada corpo de prova recebeu cinco puncionamentos para a marcação de furações na ZAC, sendo três furos a $3 \mathrm{~mm}$ de afastamento da zona de ligação afastados entre si por aproximadamente $15 \mathrm{~mm}$, assim como outros dois furos, intercalando os anteriores, a $5 \mathrm{~mm}$ de afastamento, medidos e enumerados em ordem crescente na direção da soldagem entre 50 a $100 \mathrm{~mm}$ em relação ao eixo da solda, conforme a Figura 4.

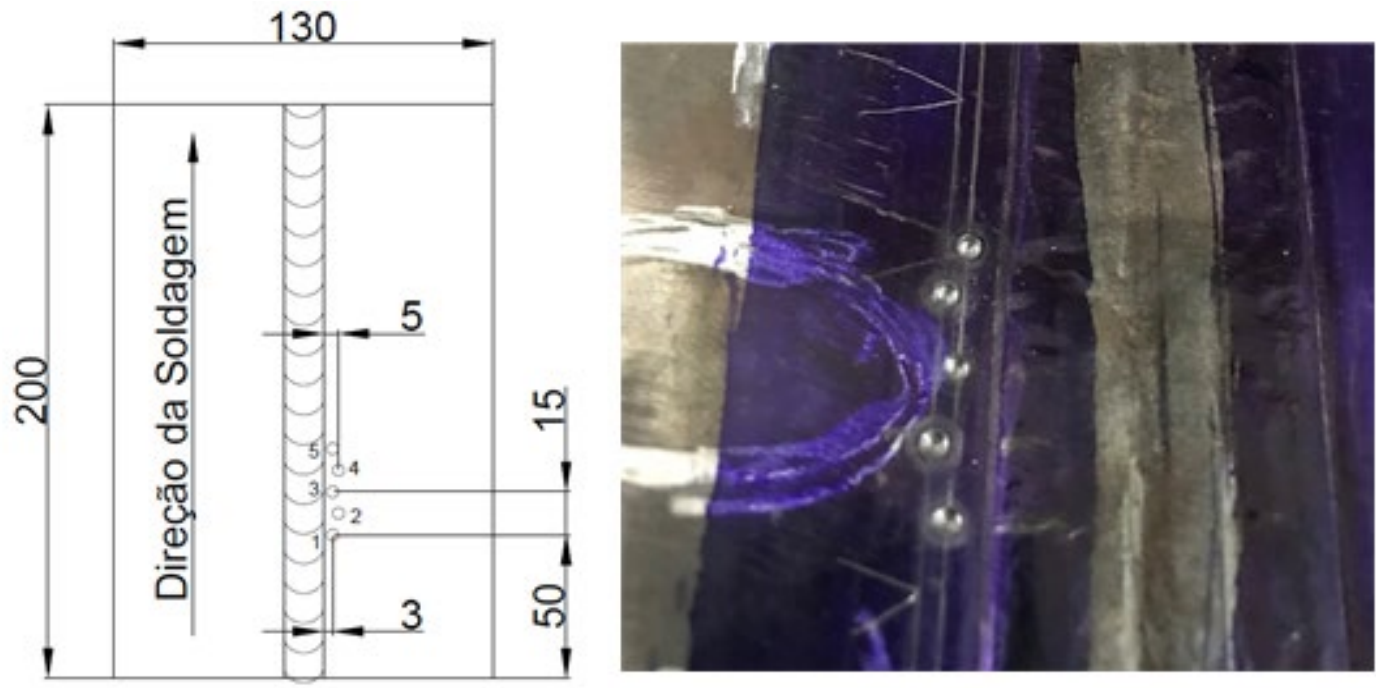

Figura 4. Marcação e medição dos pontos na ZTA.

Com o auxílio de uma furadeira de coluna foram realizadas as furações com broca de centro de diâmetro 2,0 mm a uma profundidade de aproximadamente $2 \mathrm{~mm}$. Após as furações, os centros dos furos foram mapeados $(x, y)$, em relação a uma referência posicionada na extremidade oposta das chapas. Tal mapeamento (referenciamento), determinando o posicionamento em coordenadas $(X, Y)$ dos centros dos furos, foi feito numa Máquina de Medição por Coordenadas (MMC) de controle numérico computadorizado com resolução de 0,0005 mm e certificado de calibração CCI 0132/2019.

\subsection{Tratamento Térmico para Alívio de Tensões (TTAT) e Cálculos das Tensões}

Após mapeados os furos, os corpos de teste foram submetidos a um TTAT, introduzidos no forno tipo mufla, previamente calibrado, por 60 minutos a uma temperatura de patamar de $680^{\circ} \mathrm{C}$ (abaixo da temperatura de recristalização), baseado em estudo prévio [11] e resfriado ao ar livre, em temperatura ambiente de 28 ㄷ.

Após o tratamento térmico, foram realizadas novas medições das coordenadas $(X, Y)$ dos furos, para a determinação dos deslocamentos produzidos pelo tratamento térmico. A partir da extensão dos deslocamentos dos furos, foram calculadas as tensões residuais, conforme Callister [12], utilizando os valores de módulo de elasticidade 207 GPa e coeficiente de Poisson 0,3 pelas Equações 2-4:

$\sigma_{x}=-\frac{E}{1-v^{2}}\left(\varepsilon_{x}+v \varepsilon_{y}\right)$ 
$\sigma_{y}=-\frac{E}{1-v^{2}}\left(\varepsilon_{y}+v \varepsilon_{x}\right)$

$\varepsilon=\frac{\Delta L}{L_{0}}$

Em que:

$\sigma_{x}=$ Tensão residual longitudinal (na direção do eixo $\mathrm{x}$ ) [Pa];

$\sigma_{y}=$ Tensão residual transversal (na direção do eixo y) [Pa];

$\mathrm{E}=$ Módulo de elasticidade $[\mathrm{Pa}] ;$

$\varepsilon_{x}=$ Deformação longitudinal na direção de soldagem;

$\varepsilon_{y}=$ Deformação transversal na direção de soldagem;

$v=$ Coeficiente de Poisson;

$\Delta \mathrm{L}=$ Deslocamento dos pontos [mm];

$\mathrm{L}_{0}=$ Comprimento útil do cordão de solda $[\mathrm{mm}] ;$

O cálculo das tensões residuais em diferentes pontos da junta foi baseado na extensão dos deslocamentos dos centros furos (DPC), após o tratamento térmico. Com a finalidade de redução dos erros aleatórios, neste trabalho foram utilizados três corpos de prova para cada processo e, em todos, foram utilizados o mesmo procedimento, soldador e equipamentos.

\section{Resultados e Discussões}

Os erros aleatórios existentes nas medições são provenientes, principalmente, da imprecisão na usinagem. Utilizando médias das medições foram obtidos os seguintes resultados, conforme a Tabela 3.

Tabela 3. Tensões residuais transversais médias obtidas em cada ponto.

\begin{tabular}{cccccc}
\hline Processo & Ponto & Tensão transversal (MPa) & Processo & Ponto & Tensão transversal (MPa) \\
ER & 1 & 368,88 & AT & 1 & 273,25 \\
ER & 2 & 385,92 & AT & 2 & 387,85 \\
ER & 3 & 431,31 & AT & 3 & 412,73 \\
ER & 4 & 465,98 & AT & 4 & 440,47 \\
ER & 5 & 486,63 & AT & 5 & 479,75 \\
\hline
\end{tabular}

Foi observada uma tendência de crescimento das tensões na sequência dos pontos. O ponto 1 apresentou, em média, menores níveis de tensões, ocorrendo o aumento gradativo com características similares em ambos os processos. Os dois processos obtiveram no ponto 5 (aproximadamente no centro do eixo paralelo ao cordão de solda) os maiores níveis de tensões.

O processo com arame tubular apresentou melhores resultados com relação aos níveis de tensões residuais comparados ao processo com eletrodo revestido, porém, os resultados obtidos pelo processo com eletrodos revestidos evidenciaram menores variações. Esses resultados estão de acordo com os experimentos de Lin e Chou [13], assim como Lin e Lee [14].

A partir das equações 2-4, é possível analisar as componentes nos eixos $x$ e y das tensões residuais obtidas, exibindo a média dos cinco pontos de medição na Tabela 4.

Tabela 4. Média das velocidades de soldagem, energias de soldagem, tensões residuais nas direções transversal e longitudinais ao cordão de solda.

\begin{tabular}{|c|c|c|c|c|}
\hline $\mathrm{CP}$ & $\begin{array}{c}\text { Velocidades } \\
\text { Médias [mm/min] }\end{array}$ & $\begin{array}{l}\text { Energia de soldagem média } \\
\qquad[\mathrm{KJ} / \mathrm{mm}]\end{array}$ & $\begin{array}{l}\text { Média de tensões residuais } \\
\text { transversais (бy) [Mpa] }\end{array}$ & $\begin{array}{l}\text { Média de tensões residuais } \\
\text { longitudinais }(\sigma x) \text { [Mpa] }\end{array}$ \\
\hline ER01 & 66,96 & 2.31 & 434,416 & 223,32 \\
\hline ER02 & 68,72 & 2,29 & 420,546 & 151,90 \\
\hline ER03 & 66,36 & 2.34 & 428,269 & 225,09 \\
\hline АT01 & 202,01 & 1,30 & 410,08 & 242,23 \\
\hline AT02 & 207,49 & 1,21 & 379,80 & 269,44 \\
\hline АT03 & 197,10 & 1,33 & 406,55 & 207,62 \\
\hline
\end{tabular}


O aumento é crescente nas tensões, mediante a progressão dos pontos (de 1 a 5) e a similaridade desse comportamento em todos os corpos de prova, considerando-se que o quinto ponto esteja localizado aproximadamente no centro da solda (no eixo X). Modenesi et al. [15], caracterizaram a distribuição de tensões transversais com chapas grossas em juntas de topo, com restrição de movimento, mostrando que as tensões de reação tendem a se somar às de origem térmica, resultando em maiores valores de tensões residuais transversais em relação às longitudinais, com o pico de tensão localizado no centro da chapa. No seu trabalho com chapas de 12,7 mm do mesmo aço, Mendes et al. [16], também observaram esse efeito da anisotropia $\left(\sigma_{y}>\sigma_{x}\right)$, porém não analisaram o crescimento nas tensões das extremidades para o centro do cordão. Este trabalho torna evidente que a condição de estado plano observada por Mendes et al. [16], Sugahara et al. [5], não se aplica às chapas com espessura muito superior a $12,7 \mathrm{~mm}$.

Conforme os experimentos de Gunnert [17], as tensões residuais na superfície de chapas soldadas com restrições de movimento e elevada espessura (acima de $25 \mathrm{~mm}$ ) podem atingir níveis de tensões extremamente elevados, próximos ao limite de escoamento do material. Nesses casos, é recomendada a realização de TTAT para a sua redução, melhorando, assim, a capacidade da junta em atender aos requisitos, da melhor forma para qual foi projetada.

\subsection{Efeitos da energia de soldagem}

A análise da Figura 5 mostra uma relação direta nos resultados obtidos entre as médias da energia de soldagem e as tensões residuais, podendo ser explicado pelo fato de que o aumento da energia de soldagem amplifica o volume de material afetado pelo calor e deformado plasticamente, consequentemente, aumentando as tensões residuais obtidas. A elevada espessura do metal de base, utilizando as restrições externas, ocasionou altos níveis de tensões residuais superficiais no presente experimento. Nesse aspecto, os resultados foram obtidos, conforme estudos realizados por Gunnert [17], Teng et al. [18], e Mishchenko e Scotti [19].
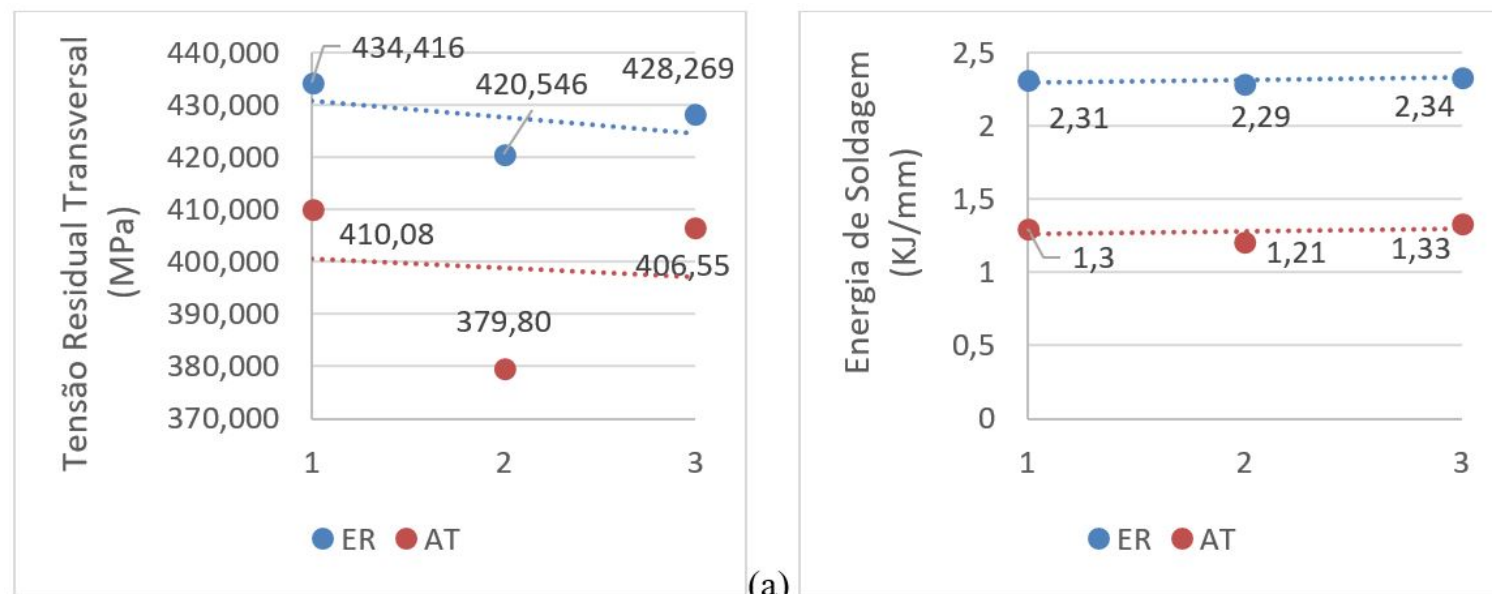

(a)

(b)

Figura 5. Tendência de tensões residuais transversais (a) e energia de soldagem (b) nos corpos de prova.

\subsection{Efeito nas tensões transversais e longitudinais}

Os processos de soldagem obtiveram um comportamento semelhante relacionado às tensões transversais e longitudinais em relação aos pontos de medição nos distintos processos, conforme Figura 6.

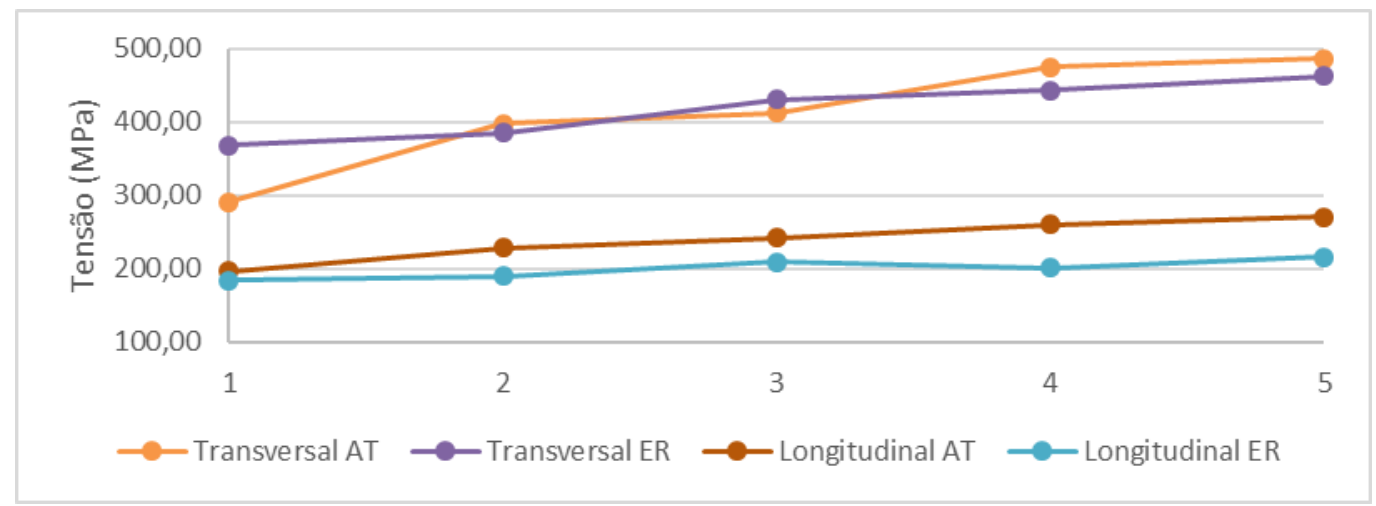

Figura 6. Tensões transversais e longitudinais nos pontos de medições nos processos ER e AT. 
Foi detectada uma maior razão de tensões transversais por longitudinais em soldas realizadas pelo processo ER. Um resultado semelhante foi obtido por Mendes et al. [16], que trabalharam com o mesmo aço na espessura de $12,7 \mathrm{~mm}$. No processo com ER, observaram-se maiores tensões residuais na direção tendendo ao eixo normal ao cordão de solda, comparado ao processo AT (Figura 7), justificando o maior valor para a razão $\sigma_{\mathrm{y}} / \sigma_{\mathrm{x}}$.

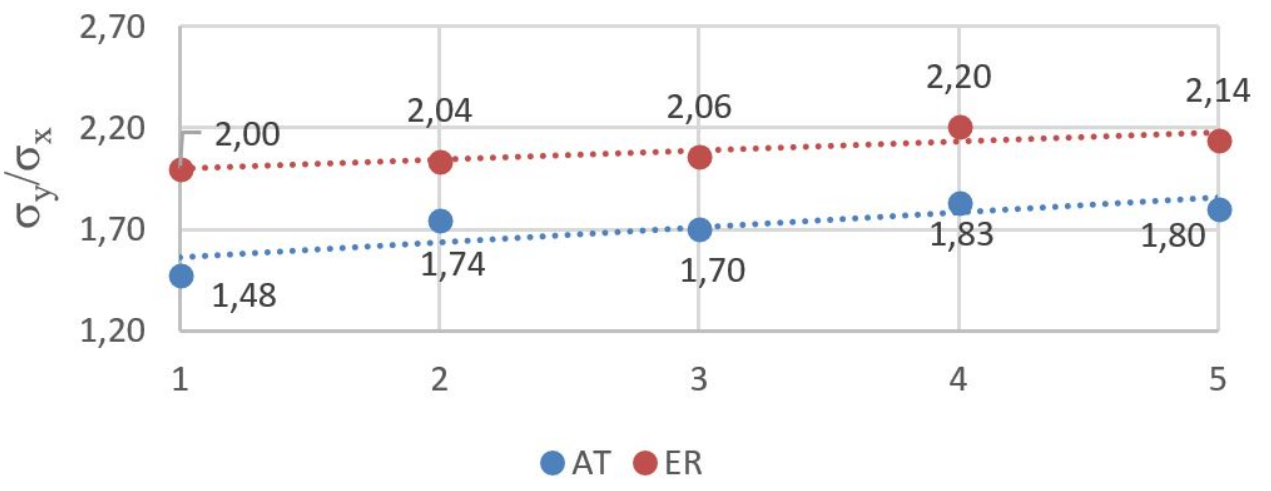

Figura 7. Tendência da constante razão das tensões transversais/longitudinais nos pontos de medição.

\subsection{Efeito relacionados à velocidade de soldagem}

Segundo Teng [20], os efeitos da velocidade de soldagem não somente interferem no volume da quantidade de material adjacente afetado pelo calor do arco, como também progressivamente reduz as tensões residuais. No presente estudo, o aumento da velocidade de soldagem ocasionou a redução nas tensões residuais em ambos os processos. Porém, no processo AT com maiores velocidades houve um efeito crescente no componente das tensões residuais longitudinais (Figura 8a), com aumento da velocidade de soldagem. Contudo, no processo ER, com menores velocidades envolvidas, não foi detectada essa variação (Figura 8b).

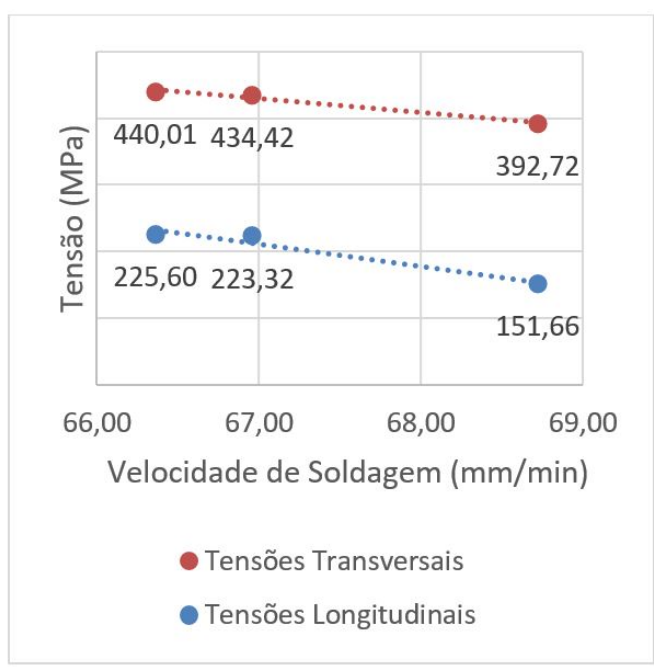

(a)

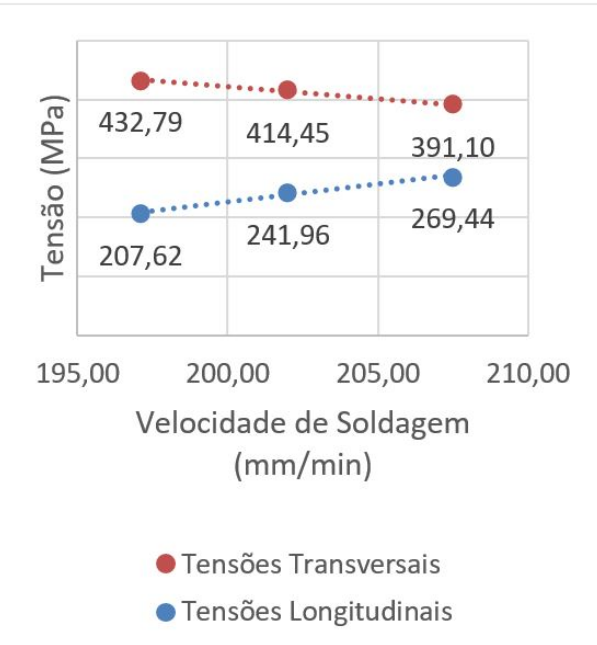

(b)

Figura 8. Relação entre tensões transversais e longitudinais com a velocidade de soldagem nos processos ER (a) e AT (b).

Analisando individualmente o processo com arame tubular, no presente experimento, com variação de velocidade de soldagem entre os corpos de prova de até 5,3\%, para níveis aproximados de energia de soldagem (Tabela 3), a tendência do efeito da velocidade de soldagem nas componentes das tensões, transversais e longitudinais, afetam diretamente a direção das tensões residuais resultantes. Conforme a Figura 9, o aumento da velocidade no processo AT apresentou uma redução na razão entre os componentes transversais $\left(\sigma_{y}\right)$ por longitudinais $\left(\sigma_{x}\right)$ das tensões. Segundo Modenesi et al. [15], o formato da poça de fusão influencia diretamente na estrutura de solidificação da zona fundida e as variações nos parâmetros, como a velocidade de soldagem, podem alterar a geometria da poça de fusão (elíptica para baixas velocidades e em gota ocorrendo em maiores velocidades). A alteração da geometria da poça de fusão tem relação com as direções das isotermas de solidificação e, consequentemente, com as direções das tensões oriundas pela contração. 


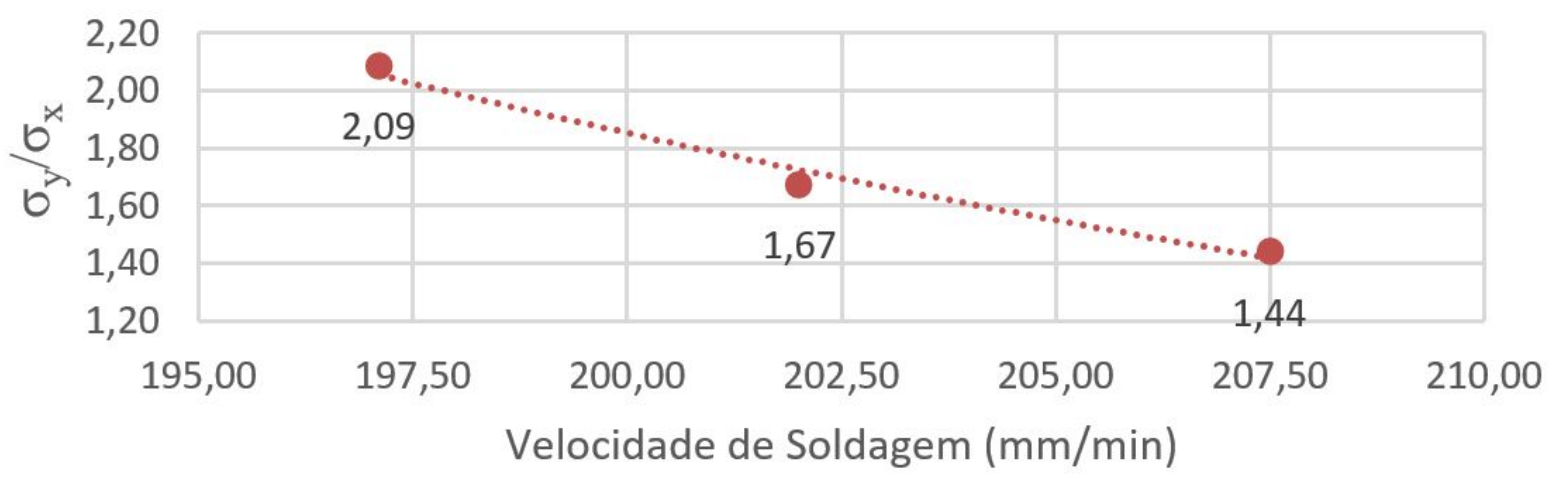

Figura 9. Efeito da velocidade de soldagem na razão das tensões transversais/longitudinais no processo AT.

\section{Conclusões}

A realização do presente estudo detectou que na soldagem, com restrição das deformações do aço ASTM 131 gr. AH36 de espessura $31,75 \mathrm{~mm}$, o processo de soldagem com arame tubular desempenhou um melhor resultado comparado ao processo com eletrodo revestido em relação aos níveis de tensões residuais provenientes da soldagem. A maior energia de soldagem, ocasionada nesse experimento pelo processo ER, por afetar um maior volume de material adjacente pelo calor, mostrou gerar maiores níveis de tensões residuais.

A tendência de crescimento nas tensões residuais, conforme sequência dos pontos, demonstra que no primeiro terço do comprimento no sentido longitudinal a soldagem há um aumento trativo nas tensões residuais transversais.

A elevada espessura do metal de base, utilizando restrições externas, ocasionou altos níveis de tensões residuais superficiais no presente experimento. Foi detectada uma maior razão entre tensões transversais por longitudinais em soldas realizadas pelo processo ER. Esse processo apresentou uma tendência maior das tensões residuais que se direcionarem perpendicularmente ao cordão de solda, comparado com o processo AT.

A velocidade de soldagem evidenciou uma tendência à redução das tensões residuais nos dois processos, porém, no processo AT foi detectado que mesmo com valores aproximados de energia de soldagem houve reduções, mas ocorreram tensões residuais, mediante o aumento da velocidade de soldagem.

Foi detectado, no presente experimento, na análise do processo de soldagem AT para as energias de soldagem aproximadas, que o aumento da velocidade de soldagem apresentou uma tendência no direcionamento das tensões residuais, nos corpos de prova com maiores velocidades de soldagem, tendo uma menor razão entre as tensões transversais por longitudinais. Esse fenômeno ocorre devido às maiores velocidades de soldagem acarretarem na formação de isotermas mais estreitas e longas na solidificação, alterando a geometria da poça de fusão e, consequentemente, as direções das tensões oriundas pela contração de resfriamento com restrição.

Os resultados encontrados reforçam a aplicabilidade do método de deslocamento de pontos coordenados (DPC) no estudo das tensões residuais como uma ferramenta simples, rápida e de baixo custo com excelentes resultados obtidos também em corpos de prova de espessura elevada, podendo sofrer adaptações de versatilidade e tornar-se utilizado em canteiros de obras e linhas de produção. Porém, a realização das marcações e furações e o tratamento térmico para alívio de tensões devem ser realizados com rígida precisão, para se obter uma boa confiabilidade dos resultados.

\section{Agradecimentos}

Os autores desse trabalho agradecem ao PPGEM da Universidade Federal de Pernambuco e ao CNPQ e CAPES pelo suporte financeiro.

\section{Referências}

[1] Macherauch E, Kloos KH. Origin, measurements and evaluation of residual stresses. In: MacHerauch E, Hauk V. Residual stresses in science and technology. Verlag: DGM inform.; 1987.

[2] Rodacoski MR. Medição de tensões residuais em holografias eletrônicas. Florianopólis: UFSC; 2002.

[3] Siqueira Filho AV, Rolim TL, Yadava YP, Cardoso FIB, Guimarães PB, Maciel TM, et al. Development of methodology for measurements of residual stresses in welded joint based on displacement of points in a coordinated table. Materials Research. 2013;16(2):322-326. http://dx.doi.org/10.1590/S1516-14392013005000001.

[4] Melo LGTC, Cardoso, Mendes CE, Ferreira RAS, Barros PS, Rolim TL. Welded joints' heat affected zone's extension prediction by switching welding parameters. Materials Research. 2017;20(Suppl 2):651-656. http://dx.doi.org/10.1590/1980-5373-mr-2016-1019. 
[5] Sugahara HJ, Barros PS, Melo LGTC, Gonçalves IL, Rolim TL, Yadava YP, et al. Measurement of Residual Stresses in Welded Joints Processed by DCP Method -. Materials Research. 2018;21(4). http://dx.doi.org/10.1590/1980-5373-mr-2017-0926.

[6] American Society for Testing and Materials. ASTM A131/A131M-19: Standard specification for structural steel for ships. West Conshohocken: ASTM International; 2019.

[7] American Welding Society. AWS A5.1/A5.1M: Specification for carbon steel electrodes for shielded metal arc welding. Miami: AWS; 2012.

[8] American Welding Society. AWS A5.20/A5.20M: Specification for Carbon Steel Electrodes for Flux Cored Arc Welding. Miami: AWS; 2015.

[9] Petrobras. N-133 N: Soldagem. Rio de Janeiro: Petrobras; 2017.

[10] American Welding Society. AWS D1.1/D1.1M: 2010: strutural welding code - Steel. Miami: AWS; 2010.

[11] Gonçalves IL. Influência da temperatura no tratamento de alívios de tensões em uma junta soldada baseado no método DPC: 2015 [dissertação mestrado]. Recife: Universidade Federal de Pernambuco; 2015.

[12] Callister WD. Ciência e engenharia de materiais: Uma introdução. 5. ed. São Paulo: LTC; 2002. p. 78-100.

[13] Lin YC, Chou CP. A new technique for reducing the residual stress induced by welding in type 304 stainless steel. Journal of Materials Processing Technology. 1995;48(1-4):693-698. http://dx.doi.org/10.1016/0924-0136(94)01710-I.

[14] Lin YC, Lee KH. Effect of preheating on the residual stress in type 304 stainless steel weldment. Journal of Materials Processing Technology. 1997;63(1-3):797-801. http://dx.doi.org/10.1016/S0924-0136(96)02727-6.

[15] Modenesi PJ, Marques PV, Santos BD. Introdução à metalurgia de soldagem. Belo Horizonte: UFMG; 2012. [acesso em 13 jun. 2019 ]. Disponível em: http://demet.eng.ufmg.br/wp-content/uploads/2012/10/metalurgia.pdf

[16] Mendes CE, Melo LGTC, Ferreira RAS, Barros PS, Rolim TL, Yadava YP. The back stress behavior study analyzed in residual stress of welded naval plates in different lamination directions and different thermal contributions. Materials Research. 2017;20(Suppl 2):722728. http://dx.doi.org/10.1590/1980-5373.

[17] Gunnert R. Method for Measuring tri-axial residual stresses. In: Doc. IIW X-184-57-OE. Commission X of the International Institute of Welding, 1958.

[18] Teng T, Fung CP, Chang PH. Effect of weld geometry and residual stresses on fatigue in butt-welded joints. International Journal of Pressure Vessels and Piping. 2002;79(7):467-482. http://dx.doi.org/10.1016/S0308-0161(02)00060-1.

[19] Mishchenko A, Scotti A. Tensões residuais em soldagem a arco: uma visão holística. Soldagem e Inspeção. 2018;23(1):93-112. http://dx.doi.org/10.1590/0104-9224/si2301.10.

[20] Teng TL, Lin CC. Effect of welding conditions on residual stress due to butt welds. International Journal of Pressure Vessels and Piping. 1998;75(12):857-864. http://dx.doi.org/10.1016/S0308-0161(98)00084-2. 